02

\title{
О природе магнитных осцилляций в высокотемпературных сверхпроводниках ҮВСО
}

\author{
() П.Д. Григорьев ${ }^{1,2,3}$, Т.И. Могилюк ${ }^{4}$, Асет Хамзаулы ${ }^{2}$ \\ ${ }^{1}$ Институт теоретической физики им. Л.Д. Ландау РАН, \\ Черноголовка, Россия \\ ${ }^{2}$ Национальный исследовательский технологический университет „МИСиС“, \\ Москва, Россия \\ ${ }^{3}$ Физический институт им. П.Н. Лебедева РАН, \\ Москва, Россия \\ ${ }^{4}$ Национальный исследовательский центр „Курчатовский институт“, \\ Москва, Россия, \\ E-mail: grigorev@itp.ac.ru \\ Поступила в Редакцию 15 апреля 2019 г. \\ В окончательной редакции 22 апреля 2019 г. \\ Принята к публикации 24 апреля 2019 г.
}

Исследованы возможные причины магнитных осцилляций с малой частотой около 2\% зоны Бриллюэна, наблюдаемые в высокотемпературных сверхпроводниках семейства ҮВСО. Предложен сценарий, когда наблюдаемая частота пропорциональна разности бислойно-расщепленных замкнутых карманов поверхности Ферми, перестроенной из-за рассеяния на вектора волны зарядовой плотности. Предложен способ экспериментального определения, какой из четырех возможных сценариев возникновения этих магнитных осцилляций реализуется в ҮВСО.

Ключевые слова: магнитные осцилляции, высокотемпературные сверхпроводники, медленные осцилляции, поверхность Ферми.

DOI: 10.21883/FTT.2019.09.48092.18N

\section{1. Введение}

Высокотемпературные сверхпроводники группы YBCO: $\mathrm{YBa}_{2} \mathrm{Cu}_{3} \mathrm{O}_{6+\delta}$ и $\mathrm{YBa}_{2} \mathrm{Cu}_{4} \mathrm{O}_{8}-$ интересны как для фундаментальных исследований механизмов высокотемпературной сверхпроводимости, так и для потенциальных приложений. Наблюдение магнитных осцилляций $\mathrm{c}$ малой частотой $F_{\alpha} \approx 530 \mathrm{~T}$ в $\mathrm{YBa}_{2} \mathrm{Cu}_{3} \mathrm{O}_{6+\delta}[1]$ дало важную информацию об их электронной структуре и вызвало активный всплеск дальнейших экспериментальных и теоретических исследований [1-9] (см. также обзоры [10-13]). Похожие магнитные осцилляции с малой частотой $F_{\alpha} \approx 660 \mathrm{~T}$ были также обнаружены в $\mathrm{YBa}_{2} \mathrm{Cu}_{4} \mathrm{O}_{8}$ [14-16].

Согласно стандартной теории магнитных квантовых осцилляций [17-19], частота этих осцилляций соответствует очень малой площади карманов поверхности Ферми (ПФ) около 2\% зоны Бриллюэна. Позднее с помощью измерений в полях до $100 \mathrm{~T}$ было обнаружено [6,7], что центральный пик фурье-образа этих осцилляций окружен двумя более слабыми пиками на частотах $F_{ \pm}=F_{\alpha} \pm \Delta F_{\alpha}$, где $\Delta F_{\alpha} \approx 90 \mathrm{~T}$. Однако ни зонные расчеты, ни измерения ARPES [20,21] (фотоэлектронная эмиссия с угловым разрешением) не предсказывают таких малых карманов ПФ. Исходная поверхность Ферми YВСО состоит из одного большого почти квадратного кармана с площадью около половины зоны Бриллюэна и соответствующей большой частоте $\sim 10^{4} \mathrm{~T}$. Для уровня легирования $p<15 \%$ возникает перестройка ПФ, возможно вызванная псевдощелью, антиферромагнитным упорядочением (AFM) и/или волной зарядовой плотности (ВЗП или CDW), в результате чего образуются четыре ферми-дуги, наблюдаемые в ARPES [20,21] и расщепленные из-за бислойной кристаллической структуры. Сами по себе эти ферми-дуги не образуют замкнутых карманов ПФ, дающих магнитные квантовые осцилляции. Волновой вектор AFM $Q_{\mathrm{AFM}}=(\pi / a, \pi / b)$, равный половине диагонали зоны Бриллюэна, соединяет концы противоположных ферми-дуг (см. рис. 1, $a$ ), что создает замкнутые электронные орбиты вдоль ПФ. Два ортогональных волновых вектора ВЗП: $Q_{\mathrm{CDW}} \approx 0.6(\pi / a, 0)$ и $0.6(0, \pi / b)$ - соединяют соседние ферми-дуги, что также позволяет образовать замкнутый карман ПФ (см. рис. $1, b)$. Наличие волны зарядовой плотности подтверждается экспериментами по рентгеновскому рассеянию [22-26], ядерному магнитному резонансу [27,28] и скорости звука [29]. Измерения коэффициентов Холла и Зеебека [30-32], обработанные по упрощенной теории без учета магнитного пробоя и сверхпроводящих вихрей, указывают на наличие электронных карманов и перестройку ПФ. Напротив, антиферромагнитный порядок, вероятно, разрушается при уровне легирования $p>0.1$.

Однако оба указанных сценария перестройки ПФ дают слишком большой замкнутый карман, с площадью около 6\% зоны Бриллюэна, и таким образом не объясняют природу наблюдаемых магнитных осцилляций. 

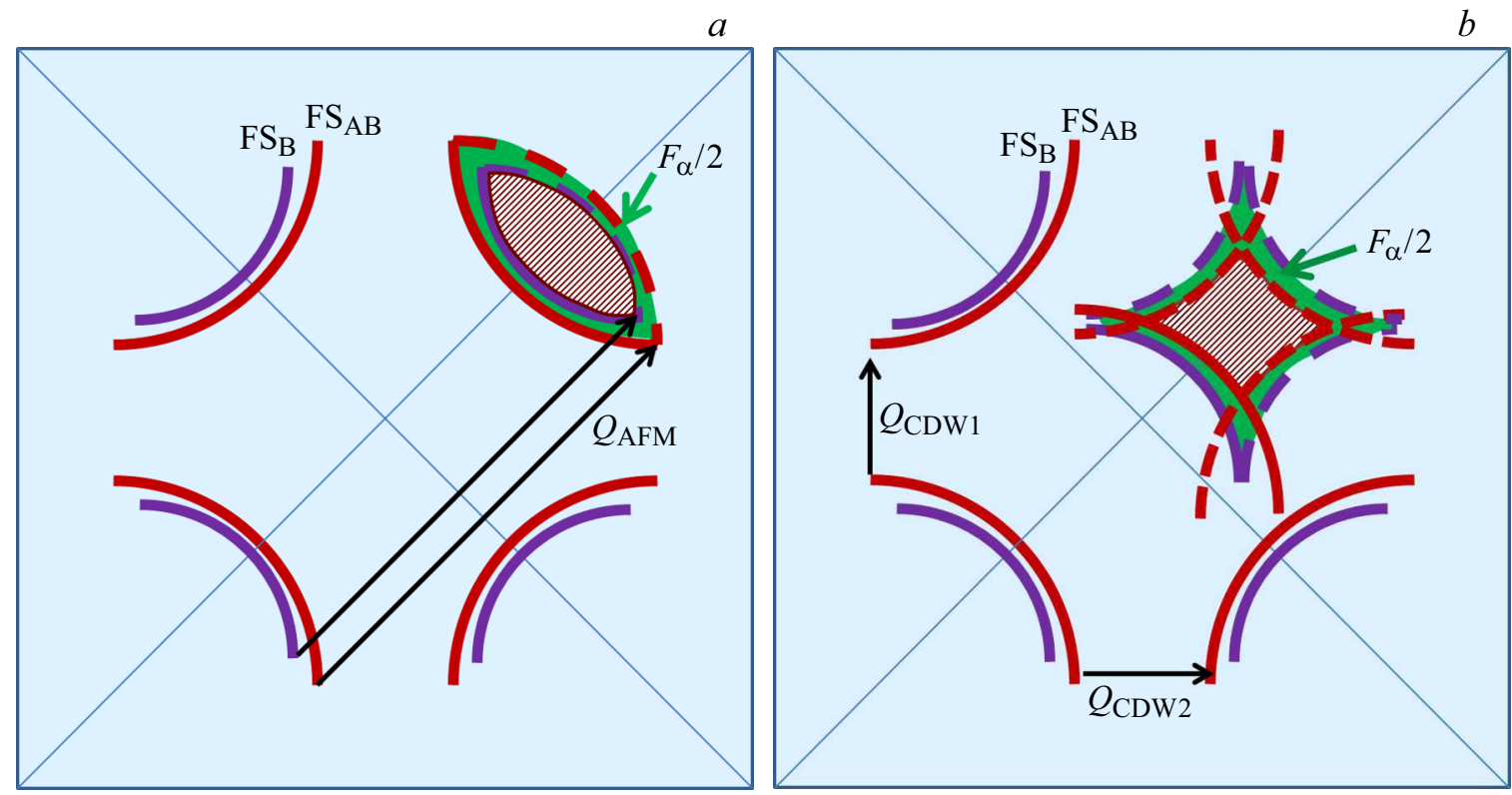

Рис. 1. Схематическая геометрическая иллюстрация бислойно расщепленных карманов поверхности Ферми (ПФ), образованных двумя различными перестройками ПФ, вызванными рассеянием на вектор $Q_{\mathrm{AFM}}=(\pi / a, \pi / b)$ антиферромагнитного упорядочения $(\mathrm{AFM})(a)$ или на двух волнах зарядовой плотности $(\mathrm{CDW})(b)$. Сами карманы заштрихованы. Разница площадей этих карманов, возникающая из-за бислойного расщепления и предположительно дающая осцилляции с частотой $F_{\alpha}$, показана сплошным фоном.

Имеются также многие другие особенности наблюдаемых магнитных осцилляций, которые трудно объяснить в рамках этих двух сценариев: (1) ровно три равноудаленных пика фурье-образа, причем амплитуда центрального пика в несколько раз больше, чем у боковых [6,7], (2) зависимость частот осцилляций от степени легирования [33] и (3) от наклона магнитного поля [6], (4) довольно хорошая устойчивость наблюдаемых осцилляций к пространственным неоднородностям и повышению температуры и др. Поэтому недавно была предложена [34,35] альтернативная интерпретация наблюдаемых магнитных осцилляций в YВСО по некоторой аналогии с эффектом магнитных осцилляций с очень малой частотой в органических металлах [36,37] и в трителлуридах редкоземельных металлов [38]. Согласно этой интерпретации, основная частота $F_{\alpha}$ соответствует не площади какого-либо кармана ПФ, а разности этих площадей, которая образуется из-за бислойного расщепления (см. рис. 1$)$. Поэтому частота $F_{\alpha}$ измеряет величину бислойного расщепления или, другими словами, интеграла перескока $t_{\perp}$ между двумя близкими слоями, а не площадь кармана ПФ. Боковые пики возникают изза электронной дисперсии поперек проводящих слоев, и разница частот $\Delta F_{\alpha}$ дает величину интеграла перескока $t_{z}$ между ближайшими бислоями.

Фурье-образ таких интерференционных или медленных осцилляций естественным образом состоит только из трех равноудаленных пиков (см. рис. 2), причем высота центрального пика более чем в 2 раза превышает высоту боковых пиков, как и наблюдается в эксперименте. При увеличении температуры Дингла или уменьшении доступного интервала магнитного поля относительная высота боковых пиков уменьшается, так что для типичных образцов $\mathrm{YBa}_{2} \mathrm{Cu}_{3} \mathrm{O}_{6+\delta}$. они различимы только в очень сильном магнитном поле выше $60 \mathrm{~T}$, в то время как центральный пик появляется при поле $>30 \mathrm{~T}$. При этом исходные частоты квантовых осцилляций, соответствующие реальным карманам ПФ, не видны в эксперименте, поскольку в отличие от медленных осцилляций сильно подавляются пространственными неоднородностями и температурой.

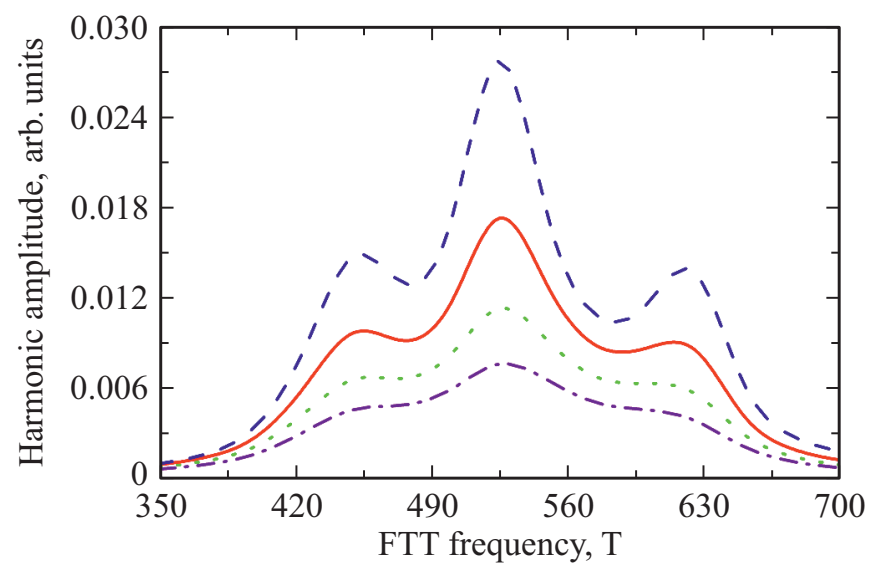

Рис. 2. Преобразование Фурье магнитных осцилляций, заданных уравнением (7) при четырех разных температурах Дингла $\pi T_{D}=\left(\hbar \omega_{c}\right)\left(B_{z}=1 \mathrm{~T}\right)=1-$ штриховая линия, $3-$ сплошная линия, 5 - пунктирная линия из точек и $7-$ штрихпунктирная линия. 


\section{2. Аналитические формулы}

Приближенная аналитическая формула для медленных осцилляций магнитосопротивления может быть получена из следующих простых рассуждений [34,35].

В двухслойных кристаллических структурах, к которым относится семейство высокотемпературных сверхпроводников ҮВСО, существует два типа межслоевых прыжков: (а) между смежными слоями, разделенными расстоянием $d$ в пределах одного бислоя, определяемый интегралом перескока $t_{\perp}=t_{\perp}\left(\mathbf{k}_{\|}\right)$и (b) между соседними эквивалентными бислоями, разделенными расстоянием $h=c^{*}-d$, определяемый интегралом перескока $t_{z}=t_{z}\left(\mathbf{k}_{\|}\right)<t_{\perp}\left(\mathbf{k}_{\|}\right)$, где $\mathbf{k}_{\|}-$импульс квазичастиц вдоль проводящего слоя. В результате спектр энергии электронов имеет вид [39]:

$$
\epsilon_{ \pm}\left(k_{z}, \mathbf{k}_{\|}\right)=\epsilon_{\|}\left(\mathbf{k}_{\|}\right) \pm \sqrt{t_{z}^{2}+t_{\perp}^{2}+2 t_{z} t_{\perp} \cos \left[k_{z} c^{*}\right]} .
$$

При $t_{z} \ll t_{\perp}$, что соответствует ҮВСО, этот энергетический спектр электронов имеет две близкие ветви, разделенные на энергию $\sim 2 t_{ \pm}\left(\mathbf{k}_{\|}\right)$

$$
\epsilon_{ \pm}\left(k_{z}, \mathbf{k}_{\|}\right) \approx \epsilon_{\|}\left(\mathbf{k}_{\|}\right) \pm t_{\perp}\left(\mathbf{k}_{\|}\right) \pm 2 t_{z}\left(\mathbf{k}_{\|}\right) \cos \left[k_{z} c^{*}\right] .
$$

Первая ветвь спектра, имеющая более низкую энергию, соответствует четной (вдоль оси $z$ ) волновой функции бислоя и в литературе называется „,bonding“. Вторая соответствует нечетной волновой функции бислоя и в литературе называется „antibonding“. Соответствующая поверхность Ферми в проводящей плоскости, схематически изображенная на рис. 1, содержит четыре расщепленные ферми-дуги, показанные красными (для bonding) и фиолетовыми (для antibonding) линиями в соответствии с данными ARPES [20,21]. Согласно стандартной интерпретации магнитных осцилляций в YBCO, наблюдаемая частота соответствует замкнутому карману ПФ, закрашенному коричневым цветом на рис. 1 и образованному из ферми-дуг в результате смещения либо на вектор AFM, как на рис. 1, $a$, либо (что на данный момент является более распространенным мнением) на два вектора ВЗП, как изображено на рис. $1, b$. В нашей интерпретации наблюдаемые осцилляции на частоте $F_{\alpha}$. соответствуют удвоенной разности этих бислойно расщепленных карманов, которая закрашена зеленым цветом на рис. 1.

Согласно (2), в ҮВСО должно быть как минимум два типа расщепления исходных частот магнитных осцилляций: большее расщепление $\Delta F_{\perp}=t_{\perp} B /\left(\hbar \omega_{c}\right)$, где $t_{\perp}=\left\langle t_{\perp}\left(\mathbf{k}_{\|}\right)\right\rangle \neq 0$ и угловые скобки означают усреднение по импульсу в плоскости $\mathbf{k}_{\|}$на ПФ, и меньшее расщепление $\Delta F_{c}=2 t_{z} B / \hbar \omega_{c} \ll \Delta F_{\perp} \ll f_{\alpha}$ из-за $k_{z}$ дисперсии электронов, где мы также предполагаем $t_{z}=\left\langle t_{z}\left(\mathbf{k}_{\|}\right)\right\rangle \neq 0$. Эти два расщепления приводят к четырем фундаментальным частотам квантовых осцилляций $F_{\beta} \pm \Delta F_{\perp} \pm \Delta f_{c}$ с близкими амплитудами. Медленные осцилляции магнитосопротивления, возникающие из-за этих четырех частот, приводят к гораздо более богатому набору частот, чем для ранее рассмотренных [36,37] однослойных структур, которые имели только две частоты $F_{\beta} \pm \Delta F_{c}$.

Металлическая проводимость вдоль $i$-й оси $\sigma_{i}$ дается суммой вкладов всех карманов $\beta$ и двух компонент спина $s$

$$
\sigma_{i}=\sum_{\beta, s} \sigma_{i, \beta, s}=\sum_{\beta, s} e^{2} g_{F \beta} D_{i, \beta} .
$$

При низкой температуре каждый карман $\beta$ вносит вклад в общую металлическую проводимость вдоль оси $i$ при низкой температуре через произведение плотности электронных состояний $g_{F, \beta}=g_{\beta}\left(\epsilon=E_{F}\right)$ и коэффициента диффузии электронов $D_{i, \beta}$. Оба вносят свой вклад в осцилляции, поскольку они изменяются в зависимости от магнитного поля $B_{z}$, перпендикулярного проводящим слоям $x-y$ как [40]

$$
\frac{g_{F \beta}}{g_{0 \beta}}=1-\sum_{l= \pm 1} 2 J_{0}\left(2 \pi \frac{\Delta F_{c}}{B_{z}}\right) \cos \left(2 \pi \frac{F_{\beta}-i \Delta F_{\perp}}{B_{z}}\right) R_{D},
$$

где $R_{D}=\exp \left(-2 \pi^{2} T_{D} /\left(\hbar \omega_{c}\right)\right)$ - фактор Дингла [41], описывающий подавление осцилляций из-за беспорядка, и $J_{0}-$ функция Бесселя. Аналогично

$$
\frac{D_{i, \beta}}{D_{0 i, \beta}}=1-B_{i, \beta} \sum_{l= \pm 1} J_{0}\left(2 \pi \frac{\Delta F_{c}}{B_{z}}\right) \cos \left(2 \pi \frac{F_{\beta}-l \Delta F_{\perp}}{B_{z}}\right) R_{D} .
$$

В общем случае коэффициент $B_{i} \neq 2$. Более того, как показывает расчет методами диаграммной техники Фейнмана [42], само выражение (5) зависит от направления $i$ : оно различается вдоль и поперек проводящих слоев, так что коэффициент $B_{i}$ может иметь слабую зависимость от магнитного поля. Подставляя (4), (5) в (3), получаем четыре типа осциллирующих слагаемых: (а) гармоники первого порядка по фактору Дингла $R_{D}$, быстро осциллирующие на частотах $\sim F_{\beta}$, (b) вторые гармоники с амплитудой $\sim R_{d}^{2}$ и частотой $\sim 2 F_{\beta}$, (c) сверхмедленно осциллирующий член

$$
\sigma_{\mathrm{USIO}}\left(B_{z}\right) \propto J_{0}^{2}\left(2 \pi \Delta F_{c} / b_{z}\right) R_{d}^{2}
$$

с частотой $\sim 2 \Delta F_{c}$ и $(\mathrm{d})$ медленно осциллирующий член с частотой $\sim 2 \Delta F_{\perp}$

$$
\sigma_{\mathrm{SIO}}\left(B_{z}\right) \propto J_{0}^{2}\left(2 \pi \frac{\Delta F_{c}}{B_{z}}\right) \cos \left(4 \pi \frac{\Delta F_{\perp}}{B_{z}}\right) R_{D}^{2} .
$$

Последние два члена, как и вторые гармоники, имеют второй порядок по $R_{D}$. Однако как было показано в работах [35-37], они (a) намного слабее подавляются температурой и $(b)$ в них входит другой фактор Дингла $R_{D}^{*}>R_{D}$. Фактор Дингла медленных (или интерференционных) осцилляций не включает макроскопические (длинноволновые) пространственные неоднородности с характерным размером больше ларморовского радиуса, которые однако сильно подавляют обычные 
квантовые осцилляции из-за размытия уровня Ферми аналогично действию температуры. Как было показано экспериментально в органических металлах [36], эффективная температура Дингла $T_{D}^{*}$ медленных осцилляций более чем в 5 раз была меньше температуры Дингла $T_{D}$ стандартных квантовых осцилляций. Поэтому эти медленно осциллирующие слагаемые обычно намного заметнее, чем исходные первые гармоники квантовых осцилляций.

Сверхмедленные осцилляции, описываемые членом (6), тоже сложно наблюдать в ҮВСО. В интервале магнитных полей 40-100Т, где обычно наблюдаются магнитные осцилляции в YВСО, их сложно выделить на фоне монотонной зависимости магнитосопротивления [43-45] из-за их малой частоты $2 \Delta F_{c} \approx 90 \mathrm{~T}$ и не периодичности, поскольку функция Бесселя $J_{0}(x)$ не периодична при $x \sim 1$. Поэтому для наблюдения этих сверхмедленных осцилляций нужны очень чистые образцы, в которых магнитные осцилляции наблюдаются уже в более слабых полях. Тем не менее такие сверхмедленные осцилляции на частоте $\sim 100$ Т были обнаружены в YBCO [9].

Намного заметнее должны быть осцилляции, описываемые последним членом (7). Их преобразование Фурье показано на рис. 2 и оказывается очень похожим на экспериментальные данные в ҮВСО $[6,7,10,12]$. Имеются три равноотстоящие гармоники. Амплитуды боковых пиков более чем в 2 раза меньше центрального и дополнительно уменьшаются с уменьшением как фактора Дингла $R_{D}$, так и интервала поля, доступного в эксперименте.

Заметим, что в пределе слабого поля $B_{z}$ и соответственно $2 \pi \Delta F_{c} / B_{z} \gg 1$ функцию Бесселя можно заменить асимптотикой для больших значений аргумента. Тогда сверхмедленные осцилляции (6) станут периодическими по $1 / B_{z}$, а медленные осцилляции (7) будут даваться выражением [34]:

$$
\begin{aligned}
& B_{i, \beta} \sum_{j, l, l^{\prime}= \pm 1} \cos \left(2 \pi \frac{2 j \Delta F_{\perp}+l\left(1-l^{\prime}\right) \Delta F_{c}}{B_{z}}\right) \\
& =2 B_{i, \beta}\left[2 \cos \left(\frac{4 \pi \Delta F_{\perp}}{B_{z}}\right)+\sum_{l= \pm 1} \cos \left(4 \pi \frac{\Delta F_{\perp}+l \Delta F_{c}}{B_{z}}\right)\right] .
\end{aligned}
$$

Первый член в квадратных скобках, соответствующий $l^{\prime}=1$, дает центральный пик, а последний член, соответствующий $l^{\prime}=-1$, дает два боковых пика. Амплитуда центрального пика в два раза больше, потому что имеются четыре различных комбинации $j, l$, дающих этот член: любые $j$ и $l$ при $l^{\prime}=1$. Напротив, каждый боковой пик задается только двумя комбинациями $j, l$ при $l^{\prime}=-1: j=l$ для правого пика и $j=-l$ для левого.

Угловая зависимость расщепления частот $2 \Delta F_{c}$, пропорциональная $t_{z}$, резко отличается от угловой зависимости $2 \Delta F_{\perp}$, связанной с бислойным расщеплением $t_{\perp}$.
Частота $2 \Delta F_{c} \propto t_{z}$ имеет сильно немонотонную зависимость от угла наклона магнитного поля $\theta$ [36]:

$$
\Delta F_{c}(\theta)=\Delta F_{c}(\theta=0) J_{0}\left(k_{F} c^{*} \tan \theta\right) / \cos \theta,
$$

где $c^{*}(=11.65 \AA$ для ҮВСО) - постоянная решетки в межслойном $z$-направлении, а $k_{F}-$ импульс Ферми. Эта угловая зависимость имеет простую геометрическую интерпретацию [46]. Она такая же как и для частоты биений квантовых осцилляций в квазидвумерных металлах [36], но сильно отличается от стандартной косинусоидальной зависимости

$$
F(\theta)=F(\theta=0) / \cos \theta,
$$

типичной для квазидвумерных металлов с почти цилиндрической ПФ и характерной также для частоты $F_{\alpha}[35]$. Поэтому угловая зависимость трех частот, получаемых из (7) находится в соответствии с экспериментальными данными из работы [6].

Отдельного изучения требует угловая зависимость амплитуды магнитных осцилляций. В случае обычных квантовых осцилляций она является немонотонной (осциллирующей) функцией наклона магнитного поля из-за зеемановского расщепления электронов - так называемый эффект спиновых нулей. Амплитуда предложенных медленных осцилляций может иметь другую угловую зависимость. На эксперименте наблюдалась зависимость, похожая на спиновые нули, но не совсем регулярная [47]. Однако она может быть связана как с настоящим эффектом спиновых нулей, так и с эффектом ложных спиновых нулей [48], возникающим из-за взаимодействия угловых и квантовых осцилляций магнетосопротивления.

Приведенные выше формулы показывают, как интерференционные медленные осцилляции появляются в магнитосопротивлении. Их возникновение в намагниченности не так очевидно, но может появится в результате учета рассеяния на примесях [35], электронэлектронного взаимодействия [49] и осцилляций химического потенциала $[50,51]{ }^{1}$

\section{3. Обсуждение}

Стандартная интерпретация магнитных осцилляций в ҮВСО предполагает, что наблюдаемая частота $F_{\alpha}$. центрального пика соответствует площади замкнутого кармана ПФ, образованного из ферми-дуг перестройкой ПФ и закрашенного коричневым цветом на рис. 1. Имеются два сценария такой перестройки ПФ: (1) со смещением на вектор $Q_{A F M}=(\pi / a, \pi / b)$ антиферромагнитного упорядочения, как на рис. $1, a$, и (2), на данный момент самое распространенное описание, со смещением на два вектора ВЗП, как изображено на рис. $1, b$. Из-за бислойного расщепления электронного спектра на

\footnotetext{
${ }^{1}$ Уравнение (9) предполагает $t_{z}\left(\mathbf{k}_{\|}\right) \approx$ const. Когда $t_{z}\left(\mathbf{k}_{\|}\right)$сильно зависит отнаправления импульса в плоскости, уравнение (9) изменяется, но остается осциллирующей функцией $k_{F} c^{*} \tan \theta[52,54]$.
} 
величину $2 t_{\perp}$ каждая частота должна быть расщеплена по крайней мере на две с одинаковой амплитудой, что не наблюдается в эксперименте. Согласно нашей интерпретации, наблюдаемые осцилляции на частоте $F_{\alpha}$ соответствуют не площади замкнутого кармана ПФ, a удвоенной разности этих бислойно расщепленных карманов, которая закрашена зеленым цветом на рис. 1. В нашей интерпретации экспериментально наблюдаемая форма осцилляций с тремя пиками на преобразовании Фурье появляется естественным образом, без дополнительных предположений. Также угловая зависимость этих частот и их зависимость от степени легирования хорошо согласуется с нашим сценарием. Тем не менее остается вопрос, какой из двух сценариев, изображенный на рис. $1, a$ или $1, b$, реализуется в ҮВСО. Хотя проще первый сценарий, основанный на перестройке ПФ изза рассеяния на антиферромагнитном порядке, в пользу второго сценария, основанного на перестройке ПФ изза ВЗП, имеется ряд серьезных аргументов. Во-первых, при уровне легирования $p>0.1$, когда наблюдаются магнитные осцилляции, антиферромагнитный порядок, вероятно, уже разрушается. Во-вторых, наличие волны зарядовой плотности подтверждается экспериментами по рентгеновскому рассеянию [22-26], ядерному магнитному резонансу $[27,28]$ и скорости звука [29]. Измерения коэффициентов Холла и Зеебека [30-32], обработанные по упрощенной теории без учета магнитного пробоя и сверхпроводящих вихрей, также указывают на наличие электронных карманов и перестройку ПФ, более близкую второму сценарию. В работах $[34,35]$ указывалось, что предложенный сценарий „медленных осцилляций“ в YВСО не сильно зависит от природы исходных замкнутых карманов ПФ. Но за основной вероятный сценарий был принят тот, что изображен на рис. $1, a$ (см. рис. 1 в статье [35]). В настоящей работе предлагается еще сценарий, изображенный выше на рис. $1, b$.

Возможность определить, какой из четырех сценариев реализуется в ҮВСО, связана с детальным количественным изучением зависимости площади карманов и их разности от уровня легирования на основе экспериментальных данных [20,21] о ПФ и ее бислойного расщепления в ҮВСО и зависимости векторов ВЗП от легирования, взятым, например, из работы [25]. Затем в каждом из четырех сценариев можно определить получаемую зависимость частоты $F_{\alpha}$ от степени легирования и сравнить с имеющимися экспериментальными данными $[4,5,33]$. Например, мы провели такие предварительные расчеты в первом стандартном сценарии. Они показывают, что площадь кармана изображенного на рис. 1, $a$ коричневым овалом оказывается $\sim 3 \%$ зоны Бриллюэна даже при уровне легирования $p=0.06$ и быстро растет с ростом легирования до $6 \%$ зоны Бриллюэна при $p=0.2$. То есть она существенно превышает площадь кармана, соответствующую наблюдаемой частоте магнитных осцилляций.

\section{Финансирование}

П.Д. Григорьев благодарит госзадание 0033-2019-0001 „Развитие теории конденсированного состояния вещества“. Т.И. Могилюк благодарит гранты РФФИ 19-02-01000, 18-32-00205, 18-02-00280 и 18-02-01022. Работа частично поддержана фондом развития теоретической физики и математики „Базис“.

\section{Конфликт интересов}

Авторы заявляют, что у них нет конфликта интересов.

\section{Список литературы}

[1] Nicolas Doiron-Leyraud, Cyril Proust, David LeBoeuf, Julien Levallois, Jean-Baptiste Bonnemaison, Ruixing Liang, D.A. Bonn, W.N. Hardy, Louis Taillefer. Nature 447, 565 (2007).

[2] Suchitra E. Sebastian, N. Harrison, E. Palm, T.P. Murphy, C.H. Mielke, Ruixing Liang, D.A. Bonn, W.N. Hardy, G.G. Lonzarich. Nature 454, 200 (2008).

[3] A. Audouard, C. Jaudet, D. Vignolles, R. Liang, D.A. Bonn, W.N. Hardy, L. Taillefer, C. Proust. Phys. Rev. Lett. 103, 157003 (2009).

[4] John Singleton, Clarina de la Cruz, R.D. McDonald, Shiliang Li, Moaz Altarawneh, Paul Goddard, Isabel Franke, Dwight Rickel, C.H. Mielke, Xin Yao, Pengcheng Dai. Phys. Rev. Lett. 104, 086403 (2010).

[5] S.E. Sebastian, N. Harrison, M.M. Altarawneh, C.H. Mielke, R. Liang, D.A. Bonn, W.N. Hardy, G.G. Lonzarich. Proc. Nat1. Acad. Sci. U.S.A. 107, 6175 (2010).

[6] Suchitra E. Sebastian, N. Harrison, P.A. Goddard, M.M. Altarawneh, C.H. Mielke, Ruixing Liang, D.A. Bonn, W.N. Hardy, O.K. Andersen, G.G. Lonzarich. Phys. Rev. B 81, 214524 (2010).

[7] Suchitra E. Sebastian, N. Harrison, Ruixing Liang, D.A. Bonn, W.N. Hardy, C.H. Mielke, G.G. Lonzarich. Phys. Rev. Lett. 108, 196403 (2012).

[8] S.E. Sebastian, N. Harrison, F.F. Balakirev, M.M. Altarawneh, P.A. Goddard, R. Liang, D.A. Bonn, W. Hardy, G.G. Lonzarich. Nature 511, 61 (2014).

[9] N. Doiron-Leyraud, S. Badoux, S. Rene de Cotret, S. Lepault, D. LeBoeuf, F. Laliberte, E. Hassinger, B.J. Ramshaw, D.A. Bonn, W.N. Hardy, R. Liang, J.-H. Park, D. Vignolles, B. Vignolle, L. Taillefer, C. Proust. Nature Commun. 6, 6034 (2015).

[10] S.E. Sebastian, N. Harrison, G.G. Lonzarich. Rep. Prog. Phys. 75, 102501 (2012).

[11] B. Vignolle, D. Vignolles, M.-H. Julien, C. Proust. Comp. Rendus Phys. 14, 39 (2013).

[12] Suchitra E. Sebastian, Cyril Proust. Ann. Rev. Condens. Matter Phys. 6, 411 (2015).

[13] S.E. Sebastian, N. Harrison, G.G. Lonzarich. Phil. Trans. R. Soc. A 369, 1687 (2011).

[14] E.A. Yelland, J. Singleton, C.H. Mielke, N. Harrison, F.F. Balakirev, B. Dabrowski, J.R. Cooper. Phys. Rev. Lett. 100, 047003 (2008).

[15] A.F. Bangura, J.D. Fletcher, A. Carrington, J. Levallois, M. Nardone, B. Vignolle, P.J. Heard, N. Doiron-Leyraud, 
D. LeBoeuf, L. Taillefer, S. Adachi, C. Proust, N.E. Hussey. Phys. Rev. Lett. 100, 047004 (2008).

[16] B.S. Tan, N. Harrison, Z. Zhu, F. Balakirev, B.J. Ramshaw, A. Srivastava, S.A. Sabok-Sayr, B. Dabrowski, G.G. Lonzarich, Suchitra E. Sebastian. Proc. Natl. Acad. Sci. U.S.A. 112, 9568 (2015).

[17] A.A. Abrikosov. Fundamentals of the theory of metals. NorthHolland (1988).

[18] D. Shoenberg. Magnetic oscillations in metals. Cambridge University Press (1984).

[19] J.M. Ziman. Principles of the Theory of Solids. Cambridge Univ. Press (1972).

[20] D. Fournier, G. Levy, Y. Pennec, J.L. McChesney, A. Bostwick, E. Rotenberg, R. Liang, W.N. Hardy, D.A. Bonn, I.S. Elfimov, A. Damascelli. Nature Phys. 6, 905 (2010).

[21] S.V. Borisenko, A.A. Kordyuk, V. Zabolotnyy, J. Geck, D. Inosov, A. Koitzsch, J. Fink, M. Knupfer, B. Buchner, V. Hinkov, C.T. Lin, B. Keimer, T. Wolf, S.G. Chiuzbaian, L. Patthey, R. Follath. Phys. Rev. Lett. 96, 117004 (2006).

[22] G. Ghiringhelli, M. Le Tacon, M. Minola, S. Blanco-Canosa, C. Mazzoli, N. Brookes, G. De Luca, A. Frano, D. Hawthorn, F. He, T. Loew, M. Moretti Sala, D.C. Peets,M. Salluzzo, E. Schierle, R. Sutarto, G.A. Sawatzky, E. Weschke, B. Keimer, L. Braicowich. Science 337, 821 (2012).

[23] J. Chang, E. Blackburn, A. Holmes, N. Christensen, J. Larsen, J. Mesot, R. Liang, D. Bonn, W. Hardy, A. Watenphul, M.V. Zimmermann, E.M. Forgan, S.M. Hayden. Nature Phys. 8, 871 (2012).

[24] A.J. Achkar, R. Sutarto, X. Mao, F. He, A. Frano, S. BlancoCanosa, M. Le Tacon, G. Ghiringhelli, L. Braicovich, M. Minola, M. Moretti Sala, C. Mazzoli, R. Liang, D.A. Bonn, W.N. Hardy, B. Keimer, G.A. Sawatzky, D.G. Hawthorn. Phys. Rev. Lett. 109, 167001 (2012).

[25] S. Blanco-Canosa, A. Frano, E. Schierle, J. Porras, T. Loew, M. Minola, M. Bluschke, E. Weschke, B. Keimer, M. Le Tacon, Phys. Rev. B 90, 054513 (2014).

[26] S. Gerber, H. Jang, H. Nojiri, S. Matsuzawa, H. Yasumura, D.A. Bonn, R. Liang, W.N. Hardy, Z. Islam, A. Mehta, S. Song, M. Sikorski, D. Stefanescu, Y. Feng, S.A. Kivelson, T.P. Devereaux, Z.-X. Shen, C.-C. Kao, W.-S. Lee, D. Zhu, J.-S. Lee. Science 350, 949 (2015); H. Jang, W.-S. Lee, H. Nojiri, S. Matsuzawa, H. Yasumura, L. Nie, A.V. Maharaj, S. Gerber, Y. Liu, A. Mehta, D.A. Bonn, R. Liang, W.N. Hardy, C.A. Burns, Z. Islam, S. Song, J. Hastings, T.P. Devereaux, Z.X. Shen, S.A. Kivelson, C.-C. Kao, D. Zhu, J.-S. Lee. PNAS 113, 14645 (2016).

[27] T. Wu, H. Mayaffre, S. Krämer, M. Horvatic, C. Berthier, W. Hardy, R. Liang, D. Bonn, M.-H. Julien. Nature (London) 477, 191 (2011).

[28] T. Wu, H. Mayaffre, S. Krämer, M. Horvatic, C. Berthier, W. Hardy, R. Liang, D. Bonn, M.-H. Julien. Nature Commun. 6, 6438 (2015).

[29] David LeBoeuf, S. Krämer, W.N. Hardy, Ruixing Liang, D.A. Bonn, C. Proust. Nature Phys. 9, 79 (2013).

[30] David LeBoeuf, Nicolas Doiron-Leyraud, Julien Levallois, R. Daou, J.-B. Bonnemaison, N.E. Hussey, L. Balicas, B.J. Ramshaw, Ruixing Liang, D.A. Bonn, W.N. Hardy, S. Adachi, Cyril Proust, Louis Taillefer. Nature 450, 533 (2007).

[31] S. Badoux, W. Tabis, F. Laliberte, G. Grissonnanche, B. Vignolle, D. Vignolles, J. Beard, D.A. Bonn, W.N. Hardy,
R. Liang, N. Doiron-Leyraud, Louis Taillefer, Cyril Proust. Nature 531, 210 (2016).

[32] J. Chang, R. Daou, Cyril Proust, David LeBoeuf, Nicolas Doiron-Leyraud, Francis Laliberte, B. Pingault, B.J. Ramshaw, Ruixing Liang, D.A. Bonn, W.N. Hardy, H. Takagi, A.B. Antunes, I. Sheikin, K. Behnia, Louis Taillefer. Phys. Rev. Lett. 104, 057005 (2010).

[33] B.J. Ramshaw, S.E. Sebastian, R.D. McDonald, James Day, B.S. Tan, Z. Zhu, J.B. Betts, Ruixing Liang, D.A. Bonn, W.N. Hardy, N. Harrison. Science 348, 317 (2015).

[34] P.D. Grigoriev, T. Ziman. JETP Lett. 106, 6, 371 (2017).

[35] P.D. Grigoriev, T. Ziman. Phys. Rev. B 96, 165110 (2017).

[36] M.V. Kartsovnik, P.D. Grigoriev, W. Biberacher, N.D. Kushch, P. Wyder. Phys. Rev. Lett. 89, 126802 (2002).

[37] P.D. Grigoriev. Phys. Rev. B 67, 144401 (2003).

[38] P.D. Grigoriev, A.A. Sinchenko, P. Lejay, A. Hadj-Azzem, J. Balay, O. Leynaud, V.N. Zverev, P. Monceau. Eur. Phys. J. B 89, 151 (2016).

[39] David Garcia-Aldea, S. Chakravarty. New J. Phys. 12, 105005 (2010).

[40] T. Champel, V.P. Mineev. Phil. Mag. B 81, 55 (2001).

[41] R.B. Dingle. Proc. Roy. Soc. A 211, 517 (1952).

[42] T.I. Mogilyuk, P.D. Grigoriev. Phys. Rev. B 98, 045118 (2018).

[43] P.D. Grigoriev. Phys. Rev. B 83, 245129 (2011).

[44] P.D. Grigoriev, M.V. Kartsovnik, W. Biberacher. Phys. Rev. B 86, 165125 (2012).

[45] P.D. Grigoriev. Phys. Rev. B 88, 054415 (2013).

[46] K. Yamaji. J. Phys. Soc. Jpn. 58, 1520 (1989).

[47] B.J. Ramshaw, B. Vignolle, J. Day, R. Liang, W.N. Hardy, C. Proust, D.A. Bonn. Nature Phys. 7, 234 (2010).

[48] P.D. Grigoriev, T.I. Mogilyuk. Phys. Rev. B 95, 195130 (2017).

[49] Y. Adamov, I.V. Gornyi, A.D. Mirlin. Phys. Rev. B 73, 045426 (2006).

[50] P. Grigoriev. JETP 92, 1090 (2001).

[51] T. Champel. Phys. Rev. B 64, 054407 (2001).

[52] M.V. Kartsovnik, V.N. Laukhin, S.I. Pesotskii, I.F. Schegolev, V.M. Yakovenko. J. Phys. 1, 2, 89 (1992).

[53] C. Bergemann, S.R. Julian, A.P. Mackenzie, S. NishiZaki, Y. Maeno. Phys. Rev. Lett. 84, 2662 (2000).

[54] P.D. Grigoriev. Phys. Rev. B 81, 205122 (2010).

Редактор Т.Н. Василевская 